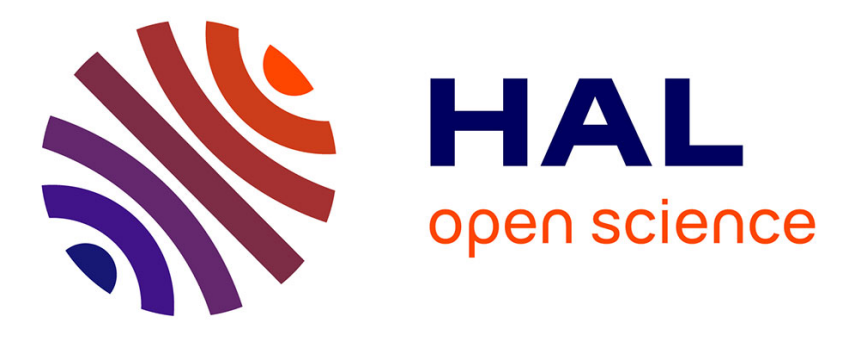

\title{
Proxy Cooperative Awareness Message: An Infrastructure-Assisted V2V Messaging
} Tomoya Kitazato, Manabu Tsukada, Hideya Ochiai, Hiroshi Esaki

\section{To cite this version:}

Tomoya Kitazato, Manabu Tsukada, Hideya Ochiai, Hiroshi Esaki. Proxy Cooperative Awareness Message: An Infrastructure-Assisted V2V Messaging. The Ninth International Conference on Mobile Computing and Ubiquitous Networking, Oct 2016, Kaiserslautern, Germany. hal-01341850

\section{HAL Id: hal-01341850 https://hal.science/hal-01341850}

Submitted on 5 Jul 2016

HAL is a multi-disciplinary open access archive for the deposit and dissemination of scientific research documents, whether they are published or not. The documents may come from teaching and research institutions in France or abroad, or from public or private research centers.
L'archive ouverte pluridisciplinaire HAL, est destinée au dépôt et à la diffusion de documents scientifiques de niveau recherche, publiés ou non, émanant des établissements d'enseignement et de recherche français ou étrangers, des laboratoires publics ou privés. 


\title{
Proxy Cooperative Awareness Message: An Infrastructure-Assisted V2V Messaging
}

\author{
Tomoya Kitazato, Manabu Tsukada, Hideya Ochiai, Hiroshi Esaki \\ Graduate School of Information Science and Technology, The University of Tokyo \\ \{pst, tsukada, jo2lxq\}@ hongo.wide.ad.jp, hiroshi@wide.ad.jp
}

\begin{abstract}
Cooperative Intelligent Transportation Systems (CITS) are being developed to make road traffic safer and more efficient. Vehicle to vehicle (V2V) communication plays an important role for the cooperative awareness of vehicles in all the CITS standards in EU, US, and Japan. Essentially the CITS application relies on the exchanged data by V2V messages (called Cooperative Awareness Message (CAM) in EU). However, the V2V communication suffers from two significant problems: 1) mixed environment and 2) obstacle interference. First, the CITS application cannot be aware of a non-CAM-aware vehicle because of low market penetration ratio of the $\mathrm{V} 2 \mathrm{~V}$ message transmitter in the early deployment phase. Second, the V2V messages may not reach the receivers because of interference by the obstacle between the vehicles. To solve these problems, the proposed system called Proxy CAM generates the necessary $\mathrm{V} 2 \mathrm{~V}$ messages in the roadside unit on behalf of sender vehicles (or non-CAM-aware vehicle) by sensing the vehicle. We design the system based on the ITS Station architecture standardized in ISO/ETSI, working with any vehicle sensing technology. Moreover, we implement the prototype system with a stereo vision for the vehicle sensing. The prototype system is evaluated in an indoor test and also validated in a field test in Tokyo with some modifications in the link layer to adapt the Japanese regulation. The results show that Proxy CAM significantly improves the coverage of $\mathrm{V} 2 \mathrm{~V}$ messaging while the system overhead is limited.
\end{abstract}

\section{INTRODUCTION}

Road transportation is one of the most important services for human mobility since ancient times. Intelligent Transportation Systems (ITS) aim at optimization of the road traffic by realizing safe, efficient and comfortable transportation. Within some research fields in ITS, Cooperative ITS, and vehicular communications became essential for the cooperation of multiple entities in the road traffic to achieve shared objectives (safety, efficiency, and comfort). Application of Cooperative ITS includes driver assistance in the near future. However, the vehicular communication also remains essential in autonomous driving to support the wider perception of the other vehicles around a vehicle that cannot be detected by the sensors equipped in the vehicle.

The International Organization for Standardization (ISO) Technical Committee 204 Working Group 16 (TC204 WG16) (also known as Communications Architecture for Land Mobile (CALM)) is developing a standard architecture for Cooperative ITS, called ITS Station reference architecture with coordination with the European Telecommunications Standards Institute (ETSI) TC ITS [1], [2]. In the US, the Institute of Electrical and Electronics Engineers (IEEE) is standardizing Wireless
Access in Vehicular Environments (WAVE) architecture in IEEE 1609 family of standards [3] as well as IEEE802.11 variant for vehicular communication as IEEE802.11p.

In these standards for Cooperative ITS, wireless one hop Vehicle-to-Vehicle $(\mathrm{V} 2 \mathrm{~V})$ broadcast is one of the fundamental techniques; that is defined as Cooperative Awareness Message (CAM) [4] in the ITS Station Architecture. The driver or the autonomous vehicle, in theory, can be aware of the other vehicle out of sight thanks to the $\mathrm{V} 2 \mathrm{~V}$ messages using wireless one-hop broadcast. However, there are potential issues of the penetration ratio of the $\mathrm{V} 2 \mathrm{~V}$ devices. To the remedy, the US National Highway Traffic Safety Administration (NHTSA) studies the possibility of regulatory work to require $\mathrm{V} 2 \mathrm{~V}$ devices in new light vehicles [5]. Despite the effort of the deployment, it is hard to imagine deploying the $\mathrm{V} 2 \mathrm{~V}$ devices at once in all the vehicles including old vehicles. Thus, we should consider the mixed environment with $\mathrm{V} 2 \mathrm{~V}$-enable vehicles and the legacy vehicles. Besides the issue of the penetration ratio, the reachability of the V2V message exchange is also key issues. The messages may be lost because of the interference of wireless radio or the blocking of obstacles such as building and large vehicles.

The objective of the paper is to solve these issues of the $\mathrm{V} 2 \mathrm{~V}$ message by the assistance of roadside infrastructure with sensors. To solve these problems, the proposed system called Proxy CAM generates the necessary V2V messages in the roadside unit on behalf of sender vehicles (or non-CAM-aware vehicle) by sensing the vehicle. We design the system based on the ITS Station architecture standardized in ISO/ETSI, working with any vehicle sensing technology. Moreover, we implement the prototype system with a stereo vision for the vehicle sensing. The prototype system is evaluated in an indoor test and also validated in a field test. The results show that Proxy CAM significantly improves the coverage of V2V messaging while the system overhead is limited.

The contributions of this work are:

- Analysis of problems of $\mathrm{V} 2 \mathrm{~V}$ communications,

- Proposal of platform for infrastructure assisted CAM for the solution

- Prototype implementation of the proposed system, and

- Experimental evaluation of prototype of infrastructure assisted CAM system.

The rest of the paper is organized as follows. Section II highlights the related works and section III analyzes the issues of $\mathrm{V} 2 \mathrm{~V}$ messages and summarizes the requirements of the 
solution. Section IV presents the system requirements and design. Section V demonstrates and evaluates our implementation. Finally, Section VI concludes our paper.

\section{RELATED WORKS}

The V2V messages exchanged among vehicles using wireless one-hop broadcast are often called "I am here" messages, which notify the sender vehicle positions and additional information. The "I am here" message using wireless one-hop $\mathrm{V} 2 \mathrm{~V}$ broadcast is standardized as CAM in EU in the ITS Station architecture. A similar concept is specified in the US as Basic Safety Message (BSM) [6] in the WAVE architecture. Japan Ministry of Land, Infrastructure, Transport and Tourism (MILT) also has the specification of the similar concept called the Advanced Safety Vehicle (ASV) V2V message.

In the ITS Station architecture, CAM is standardized in the facilities layer that provides a set of common functionalities for several ITS applications. CAM is sent to provide information of sender vehicle including time, position, motion state, and activated systems. On the reception of CAM, the information is stored in Local Dynamic Map (LDM) [7], [8] that is also standardized in the facilities layer. LDM supports various ITS applications by maintaining the information on objects of traffic entities including highly dynamic information such as vehicles, roadside, traffic conditions and accidents.

As an infrastructure assisted CITS, the Japan Metropolitan Police Department develops Driving Safety Support Systems (DSSS) for accident reduction [9]. DSSS experimented three systems: rear-end collision prevention system at entering to traffic jam which hides behind obstacles, collision prevention system at turning right at the intersection, and collision prevention system at the intersection in a bad view. The infrastructure assisted CITS using beacons and FM broadcasting are implemented on the highways in Japan.

It is analyzed that performance of Vehicular Ad-Hoc Network (VANET) depends on TX power, frequency of transmission, and the lifetime of messages in $\mathrm{V} 2 \mathrm{~V}$ communications and V2I communications [10]. Furthermore, it is known that performance of $\mathrm{V} 2 \mathrm{~V}$ messages and V2I messages strongly depends on the link quality and the propagation conditions [11]. [11] demonstrates that awareness levels for V2I communications are better than $\mathrm{V} 2 \mathrm{~V}$ communications if the roadside units are implemented on advantageous positions. [11] also explains that TX power is more important than frequency of transmission for V2X communications.

There are many ways of sensing which are useful for road traffic. Vision-based vehicle detection and tracking techniques are summarized in [12]. In vision-based technics, Millimeterwave radar and a camera are important equipment for sensing vehicles. Millimeter-wave radar can be used for measuring target range and speed. Police use Millimeter-wave radar in traffic speed regulation [12]. Millimeter-wave radar is available with a poor view in a bad weather. Stereo cameras are very effective way to sensing vehicles. Stereo cameras can detect a vehicle and, sense vehicle's position and velocity [13].
Cloud-based cooperative awareness between vehicles and pedestrians are proposed in [14]. The pedestrians send their positions to the cloud regularly from smartphones, and the cloud alerts to the vehicles in the case that the cloud finds an approach of a pedestrian to the vehicles. Vehicle-to-Pedestrian (V2P) communication is also investigated in [15]. In the work, pedestrians receive CAMs on their smartphones from invisible vehicles behind an obstacle.

\section{Problem Statement}

In this section, we explain the issues of CAM in detail. Then we analyze the design requirement for the solution.

\section{A. Issues}

1) Mixed Environment: In order to detect the vehicle using $\mathrm{CAM}$, all vehicles must be equipped with a $\mathrm{V} 2 \mathrm{~V}$ device, at least the sender device. Thus, the penetration ratio of the $\mathrm{V} 2 \mathrm{~V}$ device is the key factor for the cooperative ITS. Despite the regulatory works, it is not possible to deploy the devices to all the vehicles at once, because the legacy vehicles are already on the road. In the current assumption of CAM, these nonCAM-aware nodes are excluded and therefore their presence information are not notified to the vehicle. CAM needs to adapt to the mixed environment where CAM-supported vehicles and non-CAM-aware node (legacy vehicles, pedestrians, and bicycles) coexist.

2) Message Loss: CAM is broadcasted over an ITS-5G network within a single hop distance. The message may be lost in the delivery because the existence of obstacles between the nodes may screen the wireless radio propagation. Obstacles in the road include the building, bridge, tunnel, hill, and heavy vehicles.

Also, CAM cannot be delivered beyond the range of wireless radio propagation from the sender vehicle. This range is often referred from around 500 meters to 1000 meters using ITS-5G. The vehicle information exchange using CAM is limited to the distance of wireless radio propagation.

In the above case that CAM does not reach the receiver, the receiver is not aware of the other vehicle's presence or it is only aware of the old information. CAM needs the solution to enhance the message delivery.

\section{B. Requirements}

We design infrastructure assisted proxy system for the solution for the issues of the mixed environment. The infrastructure detects a vehicle and sends the $\mathrm{V} 2 \mathrm{~V}$ message on behalf of the vehicle. We analyze the design requirements for the system.

1) Coexistence with the CITS Standards: For the interoperability among the countries, CITS is developed based on the same architecture, protocols, and technologies. The solution must adopt the CITS standards techniques, for example, the Standard V2V messages such as CAM in EU, BSM in the US and ASV in Japan. In the ITS Station architecture, the facilities layer functionalities have particular importance because the application developed in the architecture access the function via the standard API. Thus, the solution must not require any extension to the facilities layer such as LDM. 
2) Sensor independence: The solution uses roadside sensors to obtain vehicles' data such as the position and the velocity. As we stated in section II, there are many types of sensors for this use. The solution should not depend on particular types of the sensors and be designed as a platform for infrastructure assisted $\mathrm{V} 2 \mathrm{~V}$ messages that support any kinds of sensors.

3) Real-time delivery of messages: The status of vehicles are always changing such as positions, velocities, accelerations, and the frequent transmission of $\mathrm{V} 2 \mathrm{~V}$ messages handles these changes. For example, genuine CAMs are transmitted 1 - 10 times a second. The solution should also send information about vehicles frequently. Moreover, delay in sensing and message transmission must be minimized.

4) Multiple Distributed sensors: The sensors may get a real-time data of many vehicles in the urban scenario or traffic jam. Hence, the sensors may be installed in multiple distributed locations in order to obtain wider coverage. The solution must have the capacity to handle the real-time big data from the distributed sensors.

\section{PROXY CAM}

We propose the system which solves the issues and satisfies all requirements, called Proxy CAM. We design the system compliant with CAM, however, the proposition potentially can be adapted to other V2V message standards listed in section II. The system design of Proxy CAM and the current implementation of the system are described in the section.

\section{A. System Design}

The overview of the system is shown in Fig. 1. First, the roadside sensors detect vehicles and get the information of the vehicles such as positions, velocities, and accelerations. Second, the vehicle information from the sensors is sent to the server in the infrastructure and stored in a database. Third, it generates CAMs from the data stored in the database. Finally, it broadcasts CAMs from the roadside transmitters. The CAMsupported vehicles receive these CAMs and store information into their own LDMs without any difference from the reception procedure of the genuine CAM. By this way, ITS application of the receiver vehicle can access the data from the Proxy CAM. In the following sections, we describe the system in detail by the functions.

1) Roadside Sensor: Proxy CAM uses any sensors listed in section II on a roadside to detect vehicles and to get vehicles' information. In addition to the vehicle position, speed, and acceleration, roadside sensors may take the optional data to fill the CAM format if available (i.e. lane position, steering wheel angle). The data taken from the sensors are sent to the database in the infrastructure with the vehicles' IDs. The sensor determines the vehicle's ID from an identifiable number of the vehicle if available (i.e. ID in vehicle license plate). If such identifiable number is unavailable, the sensor assigns a random ID. At the same time, the sensor assigns the same ID to the same vehicle when it identifies the same vehicle by object tracking in different times.

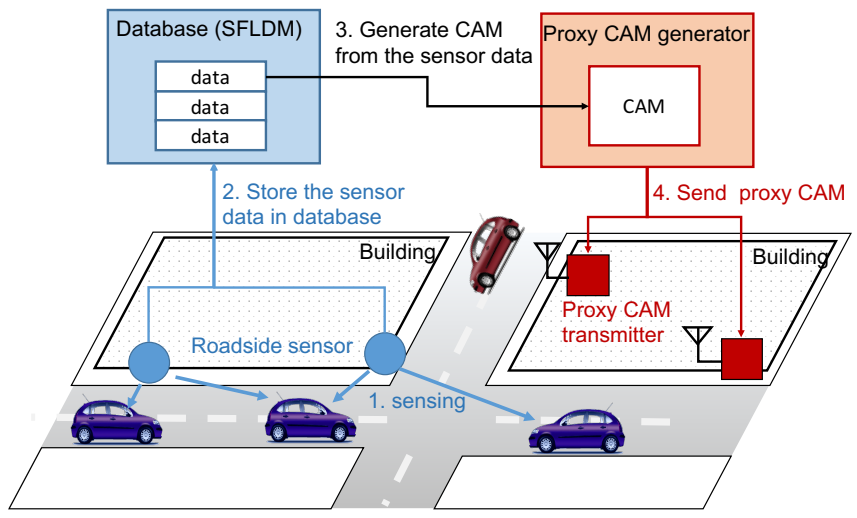

Fig. 1. System Design of Proxy CAM

2) Sensor Fusion Database: The vehicle data from the sensors are stored in a database, we name this database after Sensor Fusion Local Dynamic Map (SFLDM). SFLDM is not only a database but also a data integrator. SFLDM identifies the same vehicles data sent from multi-sensors and integrates the data by matching vehicle data such as position, speed, and heading.

3) Proxy CAM generator: Proxy CAM generator composes proxy CAMs by filling up the field of CAM from integrated data in SFLDM. The ITS PUD Header and the Generation Delta Time fields are filled with the same way as genuine CAM. In the case that the data are not available to filling up some fields, the fields remain 'unknown'. CAM has 32 bits of Station ID field which presents the sender of CAM. When the CAM generator fills up the proxy CAM field, it masks the first 24bits prefix of the Station ID field by '1'. We define the CAM with this prefix is originated from the Proxy CAM system, to distinguish from genuine CAM that does not have this prefix by the specification. The last 8 bits of the Station ID is filled by random numbers. When the same vehicle is identified, the same Station ID is used for the CAMs.

4) Proxy CAM transmitter: Proxy CAM transmitters broadcast generated proxy CAMs using the ETSI standard protocols. Proxy CAM transmitters use IEEE 802.11p as the physical access, GeoNetworking as the networking layer, and Basic Transport Protocol (BTP) as the transport layer. The installation place should be on the place where transmitters can have a good line of sight (i.e on the intersection). Moreover, the Proxy CAM system can install multiple transmitters in distributed location to cover the wider transmission range.

\section{B. System Implementation}

We implemented Proxy CAM using a stereo camera for the roadside sensor as in Fig. 2. CAM generator and CAM transmitter are combined in the same embedded Linux box (LGN-20-00) with radio equipment in this implementation. SFLDM is implemented as simple FIFO because the implementation does not have multi-sensors and does not require the data integration. 


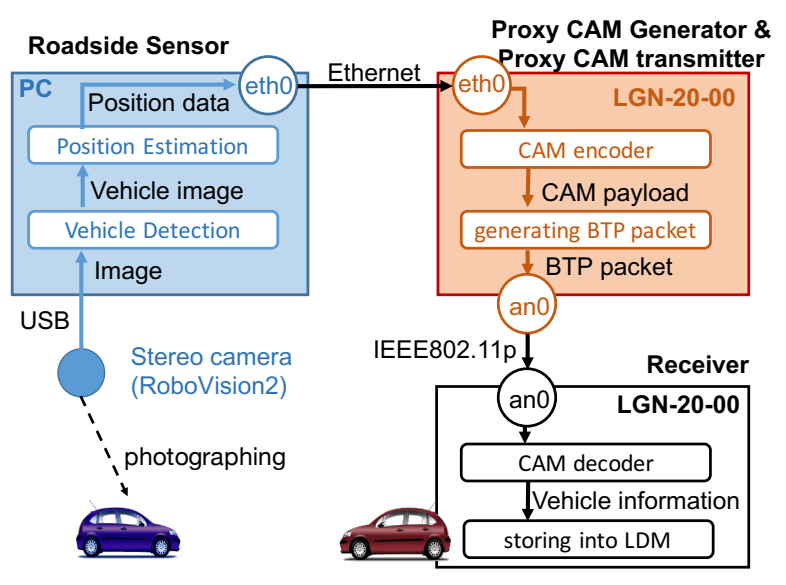

Fig. 2. Implementation Overview of Proxy CAM

1) Roadside Sensor: We used a ZMP stereo camera (RoboVision2) as a sensor. RoboVision2 can take 30 frames whose size is 1280 x 960 pixels per second. RoboVIsion2 is connected to a Windows PC via USB, and the PC detects the target vehicle with the image from the stereo camera. First, the PC detects vehicles on a road. In order to detect vehicles, we made a vehicle detector which extracts Haar-like features by a function of OpenCV. Second, the PC estimates the distance between the stereo camera and vehicles from the stereo image. We estimate the distance using block matching provided by RoboVision2 SDK. Finally, the PC can calculate the position of vehicle-based on the fixed position and angle of the stereo camera and sends 64 bits of positions ( 32 bits of latitude and 32 bits of longitude) to the CAM generator with the Ethernet using IPv4 + UDP.

2) CAM Generator: We used a Linux-based radio equipment (Commsignia LGN-20-00) for the CAM generator. The CAM generator has a reception thread which waits for data from the roadside sensor. When it receives the data, it encodes CAM with the format of the CAM standard. The CAM generator fills the data into fields of the header, the generation time, the latitude, and the longitude while it fills 'unknown' to the other fields. We use the current version of CAM in the protocol version field, and the message ID that has the prefix to distinguish the message is from Proxy CAM as described in section IV-A. The Generation Delta Time is filled with the time as the CAM specification. Station Type is filled with five which means passenger car. Latitude and longitude are filled with the values received from sensors. The total size of CAM is 54 byte including extensional filed. The CAM transmitter broadcasts the generated CAMs.

3) CAM Transmitter: The CAM transmitter is also implemented in the same Commsignia LGN-20-00 as CAM generator. The CAM transmitter broadcasts the generated CAMs with IEEE 802.11p, GeoNetworking, and BTP for the access layer, the networking layer, and the transport layer, respectively. For the GeoNetworking and BTP, we introduce CarGeo6 [16] that is an open source software of GeoNetworking. The CAM transmitter loads the generated CAMs on the payloads of BTP packets. Moreover, it transmits them by single-hop broadcast. Proxy CAM sends the message from port 2001 of BTP that is compliant to the CAM specification. We set the lifetime of CAMs for 950 milliseconds.

4) CAM Decoder: We used another Commsignia LGN-2000 for the receiver. The receiver decodes the CAMs using the functions provided by Commsignia SDK. The SDK also stores the decoded vehicle information to the LDM.

\section{Evaluation}

We evaluate Proxy CAM with the implementation described in the previous section. First, we conducted two experimental evaluations for measuring the message delivery ratio of Proxy CAM and message delay of the Proxy CAMs. The above two evaluations are done in an anechoic chamber (indoor test) to avoid the interference of IEEE 802.11p. Also, we used the anechoic chamber because IEEE $802.11 \mathrm{p}$ is prohibited to be used in outdoor in Japan. Then, we performed experimental validation and evaluation of Proxy CAM in the field test. Because of the Japanese regulation, IEEE $802.11 \mathrm{~b} / \mathrm{g}$ Ad-Hoc mode is used for the field test.

\section{A. Indoor Test : Measuring message delivery ratio}

We prepare a Linux PC to generate fake big sensor data in place of the roadside sensor. The Linux PC is connected with Ethernet to the LGN-20-00 as a CAM generator and a CAM transmitter. This Linux PC generates 1000 fake sensor data of vehicles' positions in various frequency and sends to the LGN20-00. Then, the LGN-20-00 transmitter sends the CAM using BTP + GeoNetworking to the LGN-20-00 receiver. The two LGN-20-00 were connected with IEEE 802.11p. The MCS index was 0 , the bandwidth was $10 \mathrm{MHz}$, the bitrate was 6 Mbps and the TX power was $30 \mathrm{dBm}$. Fig. 3a shows the message delivery ratio that reaches the CAM generator (via Ethernet) and to the LGN-20-00 receiver (via IEEE 802.11p). We observe that there was no loss in the frequency lower than 20 times a second in IEEE 802.11p, and there was no loss in the frequency lower than 50 times a second in Ethernet. So we see that the bottleneck of communication of Proxy CAM is in IEEE 802.11p. Proxy CAM can manage 20 data a second. GPS update positions of vehicles once a second. In this experiment, we measured the performance of the situation when only one transmitter is used. We consider that if multitransmitters are used, the performance will be improved. Then we need to consider the strategy of CAM transmission, for example, which channel will we use, how to decide the order of information to broadcast, the coverages of each transmitter.

\section{B. Indoor Test : Measuring Delay}

Proxy CAM must be real-time because the status of vehicles is always changing. Thus, we evaluate messaging delay of Proxy CAM. We measure the time from vehicle detection to reception of CAMs in the receiver. To break down the delay, we separate the total delay into two parts and measure them separately. One is the delay in the roadside sensor, and 


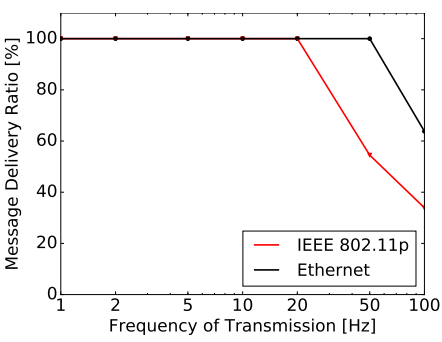

(a) Message Delivery Ratio

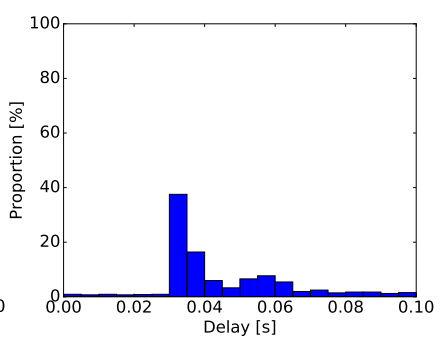

(b) Message Delay
Fig. 3. Indoor Test: Result

the other is the delay in the CAM generator and the CAM transmitter.

First, we explain the measurement of delay in the roadside sensor. We use a stereo camera and a Windows PC for a roadside sensor. The PC has Intel core i7 CPU and NVIDIA GTX980M GPU for image processing. The PC detects vehicles with CPU and estimates the position with the CPU and the GPU. We define the delay in the roadside sensor as the time between acquisition of a frame and the end of calculation of the position of the vehicle. We test for 1000 frames. As a result, it constantly takes about 0.12 seconds for the image processing per a frame in the roadside sensor.

Second, we explain the measurement of delay in the CAM generator and the CAM transmitter in the anechoic chamber. We prepare a Linux PC to generate fake data in place of the roadside sensor. This time, the Linux PC is connected to both the transmitter LGN-20-00 and the receiver LGN-20-00 with Ethernet. Then the Linux PC generated a same 8 bytes data to both the transmitter and the receiver at the same time. The receiver measures the time gap between the reception of the data from the Linux PC and the reception of CAM from the transmitter. The two LGN-20-00 were connected with IEEE 802.11p as same wireless configuration as the previous section. We tested 1000 times, and the results are shown in Fig. 3b. Fig. 3b shows that delay in the CAM generator and the CAM transmitter varied widely. The maximum delay is about 0.1 seconds, and this means the maximum of total delay (with the delay of image processing) in Proxy CAM is about 0.22 seconds. This value is small enough to use for CITS applications like collision prevention. For example, a vehicle which driving 60 kilometers an hour per hour will proceed about only 3 meters.

\section{Field Test}

In the field test, we validated that Proxy CAM can send information of non-CAM-aware vehicles and that Proxy CAM can transmit messages to dead space. And we evaluated the performance of Proxy CAM with IEEE 802.11b/g. We perform the experimental evaluation at Hongo campus of The University of Tokyo. The installation of the Proxy CAM implementation is shown in Fig. 4. We installed RoboVision2 as a roadside sensor at the intersection; that detects the target vehicle is approaching the intersection. Then the image pro- cessing Windows PC calculates the position of the vehicle. The LGN-20-00 transmitter also locates at the same intersection. It receives the data of the vehicle position from the Windows PC and broadcasts a CAM. Then the LGN-20-00 receiver on the other road receives the message from the Proxy CAM.

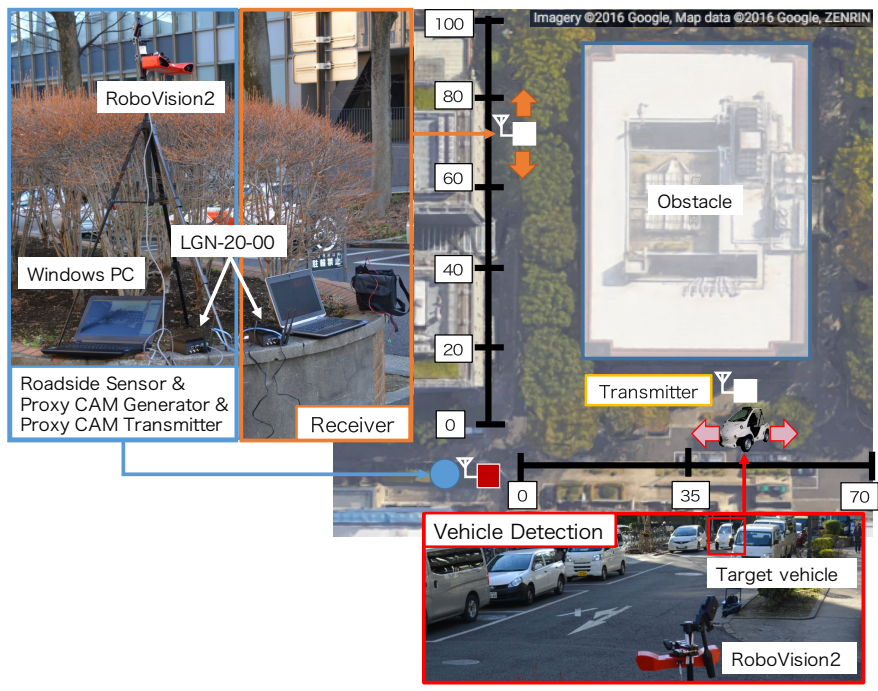

Fig. 4. Test Environment of Field Test

First, we measured the range of detection area of the sensor. As a result, the sensor can detect the vehicles as far as 70 meters. Then, we measured message delivery ratio from the sensor to the receiver by putting the LGN-20-00 transmitter and the LGN-20-00 receiver side by side as shown the left picture in Fig. 4. The CAM transmitter sends 1000 CAMs to the receiver in various frequency by IEEE $802.11 \mathrm{~b} / \mathrm{g}$ with TX power was $13 \mathrm{dBm}$; then we counted how many CAMs reached the receiver. Fig. 5a shows the result. About $90 \%$ of CAMs reached the receiver when the frequency of transmission is less than 20 times a second. And we see that delivery ratio largely decreased when the frequency is more than 20 times a second.

Then, the transmitter moves at the places where the distances from the intersection are 0,35 , or 70 meters. And the receiver is installed at the places where the distances from the intersection are $0,20,30$, or 40 until 100 meters. We sent 1000 CAMs as frequent as ten times a second and measured how many CAMs reached the receiver in all the combinations. Fig. 5b shows the results of this experiment. The horizontal axis shows the distance between the receiver and the intersection. The vertical axis shows the reception ratio of CAMs from the transmitter which locates $0,35,70$ meters away from the intersection. The lines of CAM $(35 \mathrm{~m})$ and $C A M(70 m)$ represent the case of the CAM-supported vehicle approaching the intersection while the line of Proxy CAM (Om) represents the case that the proposed system is installed in the intersection. The results show that delivery ratio is good when sender locates on the intersection because of its line of sight. However, the delivery ratio degrades, when both the transmitter and the receiver locates far from the intersection 

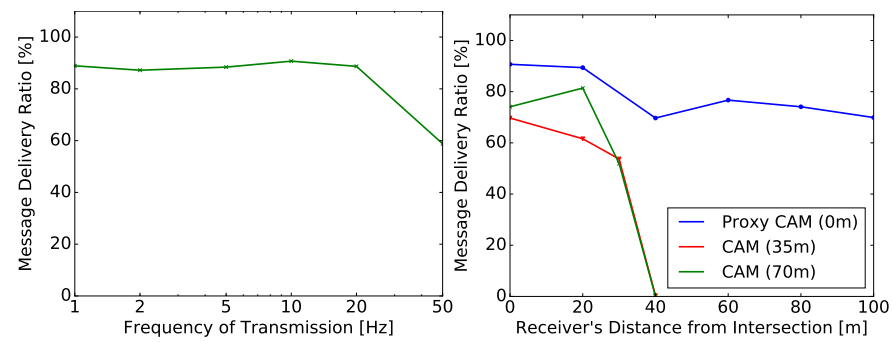

(a) Various Frequency of Transmission(b) Various Distances from The Intersection

Fig. 5. Field Test: Result

more than 30 meters. We can see that Proxy CAM is useful if its CAM transmitter is at an intersection.

We will consider the mixed environment problem described in section III with the results of field tests. If Proxy CAM does not exist in the intersection, the receiver cannot discover the approaching vehicles before entering the intersection. The result of the experimental evaluation suggests that thanks to Proxy CAM, a CAM-supported vehicle can be aware of the approaching vehicle 100 meters away from the intersection. The CAM-supported vehicles can stop before the intersection with the warning because the stopping distance of a vehicle which drives 60 kilometers an hour is 44 meters. Proxy CAM makes traffic safer in a mixed environment. Proxy CAM also solves the obstacles' interference problem. Assume the situation where two CAM-supported vehicles are approaching the intersection. One is on the priority road, and another is on the non-priority road. When obstacles interfere with V2V communication, vehicles are aware of each other 30 meters away from the intersection without Proxy CAM. Thanks to Proxy CAM, the vehicles are aware of each other 70 meters away from the intersection. As 70 meters is longer than the stopping distance of a vehicle which drives 60 kilometers, the vehicle can be aware of the other approaching vehicle before entering the stopping distance from the intersection. Thus, Proxy CAM can help road safety in a dangerous intersection.

\section{CONCLUSion AND Future Works}

We proposed the system named Proxy CAM, which generated CAMs from roadside sensors data and broadcasted CAMs with roadside transmitters to the problems of $\mathrm{V} 2 \mathrm{~V}$ messages. Then we implemented the prototype of Proxy CAM with a stereo camera. We validated the function of the prototype of Proxy CAM and evaluated its performance. We measured the message delivery ratio with various frequency and message delay of this system. As a result, the system could send 20 CAMs a second, and the maximum delay in this system was 0.22 seconds. These results show the usefulness of Proxy CAM. CITS applications perform better as the amount of information which the application uses become bigger. CITS will make road traffic safer and more efficient by utilizing Proxy CAM.

We have four future works. First, the evaluation is necessary for Proxy CAM that has multiple sensors and multiple transmitters. This requires the development of a database for storing and processing sensor data. Second, Proxy CAM needs the algorithm of message dissemination strategy (frequency, message order, and determination area) for multi-transmitter scenarios. Third, the proposed system can potentially be adapted to the other V2V standards. Thus, we need to clarify the details. Fourth, the system must be aware of the V2V message capability of the target vehicle for the proxy message transmission. The system estimates the original message delivery area and it transmits the proxy message to the wider area that the original message does not reach.

\section{ACKNOWLEDGMENT}

The authors would like to thank Tao Ye and Patrick Yang for their help of the field tests and various comments on the paper.

\section{REFERENCES}

[1] ISO 21217:2010 Intelligent transport systems - Communications access for land mobiles (CALM) - Architecture, April 2010.

[2] Intelligent Transport Systems (ITS); Communications Architecture, September 2010. ETSI EN 302665 V1.1.1 (2010-09).

[3] IEEE 1609.0 Draft Standard for Wireless Access in Vehicular Environments (WAVE) - Architecture, April 2010.

[4] Intelligent Transport Systems (ITS); Vehicular Communications; Basic Set of Applications; Part 2: Specification of Cooperative Awareness Basic Service, December 2014. ETSI EN 302 637-2 V1.3.2 (2014-11).

[5] U.S. Department of Transportation, National Highway Traffic Safety Administration. Vehicle-to-Vehicle Communications: Readiness of V2V Technology for Application. pages 1-327, August 2014.

[6] J2735: Dedicated Short Range Communications (DSRC) Message Set Dictionary, November 2009.

[7] Intelligent transport systems - Cooperative systems - Definition of a global concept for Local Dynamic Maps, November 2014. ISO/PRF TS 18750.

[8] Intelligent Transport Systems (ITS); Vehicular Communications; Basic Set of Applications; Local Dynamic Map (LDM), September 2014. ETSI EN 302895 V1.1.1 (2014-09).

[9] Nakagawa Atsushi, Nakano Tsuyoshi, and Okamoto Yasukazu. Demonstration Experiments of Driving Safety Support Systems Using Vehicleto-Infrastructure Communications Systems (Japanese), 2009.

[10] Martijn Van Eenennaam, Wouter Klein Wolterink, Georgios Karagiannis, and Geert Heijenk. Exploring the solution space of beaconing in vanets. In Vehicular Networking Conference (VNC), 2009 IEEE, pages 1-8. IEEE, 2009.

[11] Mate Boban and Pedro M. d'Orey. Exploring the practical limits of cooperative awareness in vehicular communications. March 2015.

[12] S. Sivaraman and M. M. Trivedi. Looking at vehicles on the road: A survey of vision-based vehicle detection, tracking, and behavior analysis. 14(4):1773-1795, December 2013.

[13] M. Bertozzi, A. Broggi, A. Fascioli, and S. Nichele. Stereo visionbased vehicle detection. In Intelligent Vehicles Symposium, 2000. IV 2000. Proceedings of the IEEE, pages 39-44, 2000.

[14] M. Bagheri, M. Siekkinen, and J. K. Nurminen. Cellular-based vehicle to pedestrian ( $\mathrm{v} 2 \mathrm{p}$ ) adaptive communication for collision avoidance. In Connected Vehicles and Expo (ICCVE), 2014 International Conference on, pages 450-456, November 2014.

[15] P. Merdrignac, O. Shagdar, I. B. Jemaa, and F. Nashashibi. Study on perception and communication systems for safety of vulnerable road users. In Intelligent Transportation Systems (ITSC), 2015 IEEE 18th International Conference on, pages 1876-1881, September 2015.

[16] T. Toukabri, M. Tsukada, T. Ernst, and L. Bettaieb. Experimental evaluation of an open source implementation of IPv6 geonetworking in vanets. In ITS Telecommunications (ITST), 2011 11th International Conference on, pages 237-245, August 2011. 